\title{
A Live Interior: Environments, Assemblies, Materialities
}

\author{
By Ulrika Karlsson *, Cecilia Lundbäck ${ }^{ \pm}$, Daniel Norell ${ }^{\ddagger}$, \\ Einar Rodhe $e^{+}$\& Veronica Skeppe ${ }^{\S}$
}

\begin{abstract}
This paper examines the interior as a condition that is continuously in production through the arrangement of objects and furniture. This is done along two lines of inquiry. First by examining a few different historical and contemporary conceptions of the domestic interior through the lens of architectural representation. Second by using the technique of laser scanning to document a number of inhabited interiors in two apartment buildings. Through a series of representations, or cloud drawings, produced from the scans, the paper presents three ways of reading the interior: as environments, as assemblies, and as materialities. Departing from Robin Evans' writing on drawing techniques for representing the interior and their correlation to ways of inhabitation, the paper poses questions around how the understanding of the interior may shift when using emerging techniques for architectural representation. Through readings of Walter Benjamin as well as Sylvia Lavin, the paper discusses such shifts in relation to changes in the conception of the interior and the objects that it contains.
\end{abstract}

\section{Introduction}

The domestic interior can be understood as a live environment, shaped by elements of varying temporality. Wallpaper, baseboards, mouldings, and flooring typically have a shorter duration than the architectural framework that supports them. Furniture as well as textiles, plants and the myriad of smaller items that occupy desks and shelves are rearranged in a continuous negotiation between the interior and its inhabitants. Overall, this makes the domestic interior an unstable condition, constantly in production.

Domestic interiors present a specific set of issues for architectural representation. While the exterior of a building often can be captured in its totality in a set of elevations, a void inside it, such as a room, typically becomes fractured by plan and section cuts that slice through it. Further, unlike the exterior, the interior mediates between architecture and human habitation through accommodation of everyday domestic life. A floor plan, for example, has in this sense been characterized by Pier Vittorio Aureli as a "concrete abstraction", since it "translates many determinations - money, measures, code, gender, class, rituals,

\footnotetext{
*Professor, School of Architecture, KTH Royal Institute of Technology, Sweden.

${ }^{ \pm}$Lecturer, School of Architecture, KTH Royal Institute of Technology, Sweden.

${ }^{\dagger}$ Senior Lecturer, Department of Architecture and Civil Engineering, Chalmers University of Technology, Sweden.

${ }^{+}$Senior Lecturer, Konstfack, College of Arts, Crafts and Design, Sweden.

${ }^{\S}$ Research Engineer, School of Architecture, KTH Royal Institute of Technology, Sweden.
} 
beliefs, ideologies, environmental conditions, etc. - into a specific spatial layout."1 Yet, as life in the interior is played out across scales - from rooms, to furniture, to objects - much of it often eludes being addressed in the abstracted language of architectural drawing. This is an issue of medium specificity as well as of disciplinary boundaries. Drawing has historically been conditioned by the practice of outlining, of drawing contours with lines, which promotes engagement with some properties of surfaces and objects while downplaying others. In addition, what becomes included or excluded in a drawing is dependent on professional responsibilities, belonging to fields such as architecture, interior architecture, furniture design, or industrial design.

By surveying contemporary domestic scenes in drawings, the research project Interiors Matter: A Live Interior examines the interior as an unstable condition, continuously produced through the arrangement of objects and furniture (Figure 1). The project explores laser scanning as a specific means of documentation of everyday domestic interiors and as a medium of drawing. This is done through the disciplinary lens of architectural representation, as well as by looking at different conceptions of the domestic interior and the objects that it may contain. In summary, this paper and the drawings contained in it, raise two questions: How is instability affecting the production of an interior environment? How can aspects of an interior's instability be captured, documented, or replicated using point clouds obtained through laser scanning?

The paper is structured as follows. Background and relevant literature are introduced in two sections. First, Representation and Inhabitation outlines how the historical development of techniques for representing the interior can be paralleled with the development of spatial practices of inhabitation. Second, From Collection to Stuff outlines how the interior can be understood through the organization of furniture and objects that it contains. The following section, Capturing the Live Interior, introduces $3 \mathrm{~d}$-scanning as a method as well as a spatial practice and describes how the interiors studied in the project were documented through a combination of laser scanning and photography. Results and findings are explored in two sections. Cloud Drawings focuses on the particularities of point clouds as a medium of representation, and Environments, Assemblies, Materialities discusses cloud drawings in relation to these three ways of reading the interior. Finally, the Conclusions section discusses how the cloud drawings developed in the project can open for new ways of understanding and conceptualizing the interior

1. P. V. Aureli, “Life, Abstracted: Notes on the Floor Plan”, E-flux Journal ( 2017) Retrieved from: https://www.e-flux.com/architecture/representation/159199/life-abstracted-notes-on-the-floorplan/ [Accessed 26 October 2020]. 


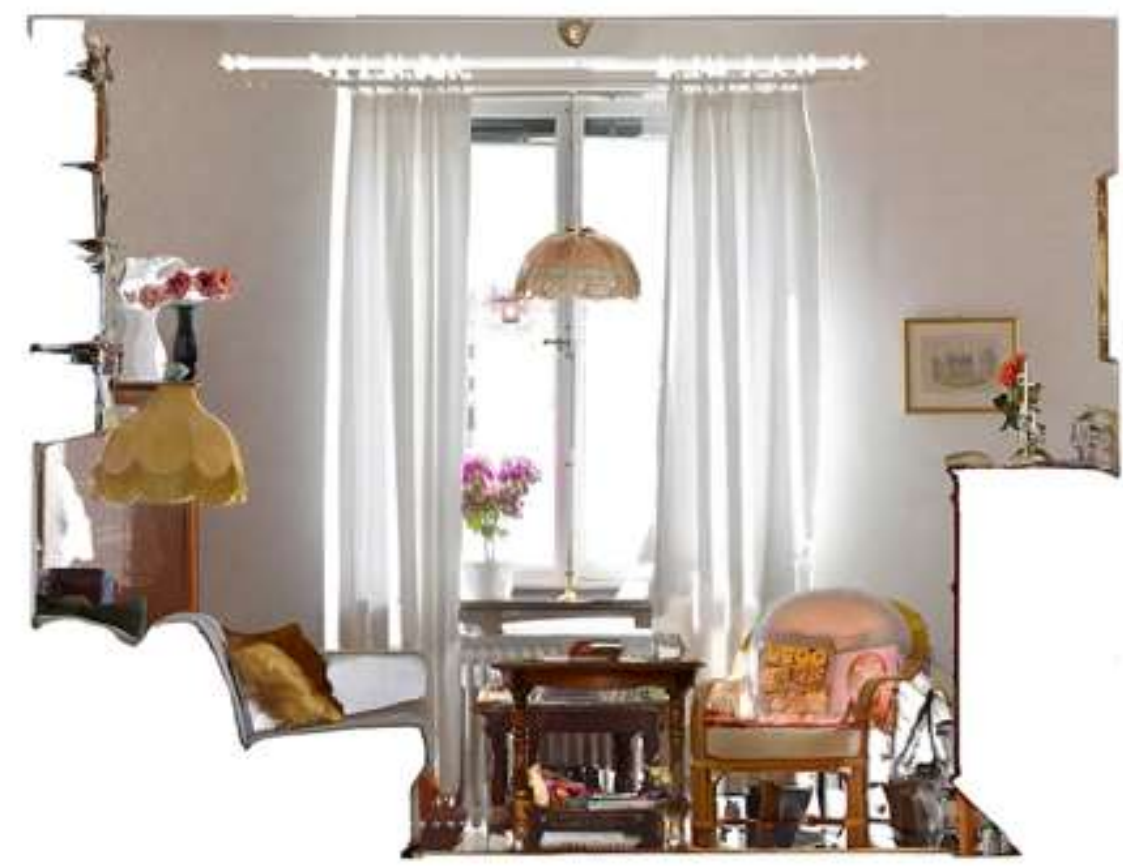

Figure 1. Interiors Matter: A Live Interior. Section view of point cloud acquired through laser scanning of a room in an apartment, Stockholm, 2019. Equal weight is assigned to ceiling, walls, floor and interior belongings. Parts of the room are invisible to the scanner and appears as white gaps or "drop shadows" behind the vase and the curtain rod.

Source: Authors.

\section{Representation and Inhabitation}

The historical development of techniques for representing the interior can be paralleled with the development of spatial practices of inhabitation. Charles Rice has described this as a "doubleness" in how the interior was conceived and developed. ${ }^{2}$ The notion of the interior emerged both in its physical presence and use as well as in the two-dimensional representations of interiors. Over time, representational techniques and spatial conditions "emerge, develop, transform and are abandoned together" and in so doing, continuously produce different conceptions of the interior. ${ }^{3}$

2. C. Rice, The Emergence of the Interior: Architecture, Modernity, Domesticity (London and New York: Routledge, 2007), 20.

3. Ibid, 26. 
A well-known reference relating modes of architectural drawing to changes in social life is Robin Evans' "The Developed Surface: An Enquiry into the Brief Life of an Eighteenth-Century Drawing Technique."4 In England during the 18th century it was common practice that interiors of palaces and villas were described in section drawings that sliced through the building in its entirety. While these sections were highly detailed, containing wall coverings, colour schemes, and décor, they emphasised the relation between rooms rather than giving a complete description of any one room. During this period, architects such as the Adam brothers developed a new kind of drawing, referred to by Evans as the "developed surface interior", or "room portrait." The room portrait showed all wall elevations as if unfolded from the plan, so that the surfaces that delimited a room, with the exception of the ceiling, were described in five discontinuous but aligned planes (Figure 2). The developed surface drawings of each room emphasized the individuality of each single space, through consistency in style, ornaments and furnishing. The use of this drawing technique was paralleled in the development of space plan organisations where the single room was increasingly detached from its situation in the larger building and became "a totally encompassing enterprise." Floor plans focused less on hierarchical relationships and the sequence of rooms, but instead introduced difference through the shape of each room.

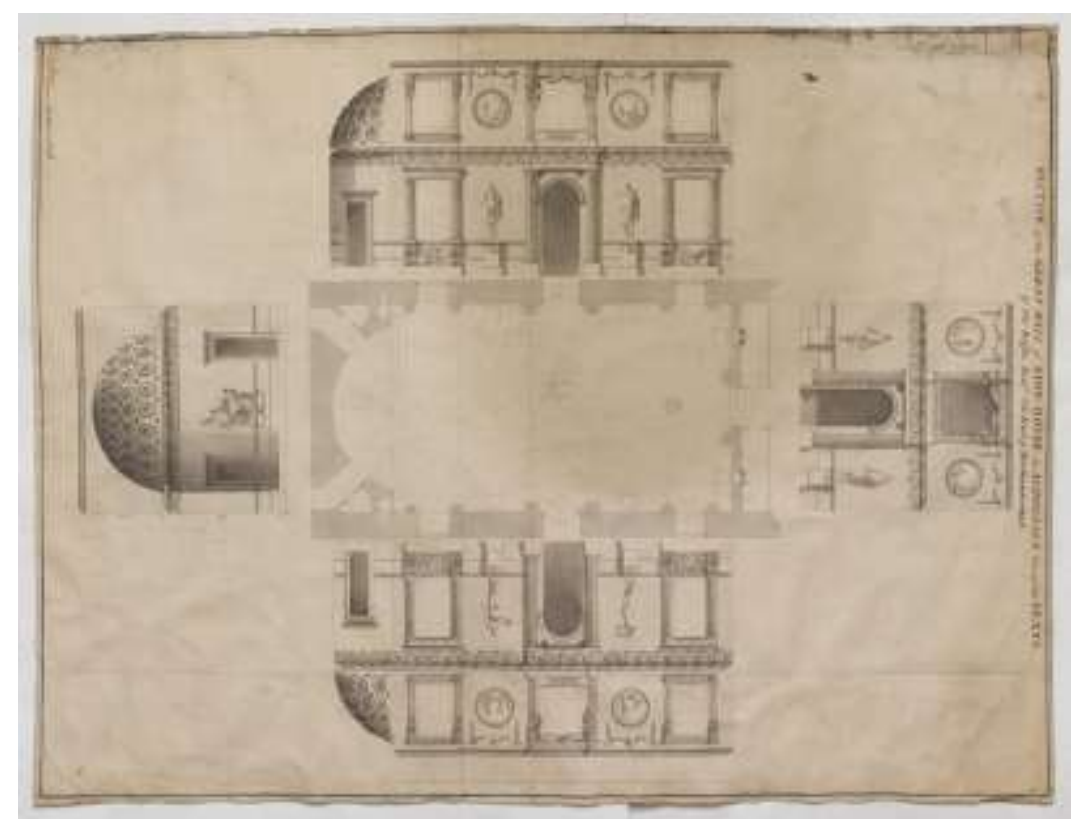

Figure 2. "Section" of the Great Hall at Syon House, by Robert Adam, 1761. A room portrait consisting of one plan and four unfolded elevations.

Source: (C) Sir John Soane's Museum, London.

4. R. Evans, "The Developed Surface: An Enquiry into the Brief Life of an EighteenthCentury Drawing Technique," in Translations from Drawing to Building (Cambridge: The MIT Press, [1989] 1997), 194-231.

5. Ibid, 202-204.

6. Ibid, 207. 
The room portrait was not just one of many unbiased ways of describing an interior, but an architectural proposition in itself. It suggested an approach to the design of interiors where articulation was pushed to the periphery of the room, so that any freestanding objects would neatly fall into one of the elevations of the walls. In addition, Evans parallels the developed surface drawing's focus on the edges of the room with social customs and habits of the time. Inhabitants occupied rooms along their walls, often accommodated by a ring of chairs. When furniture became easier to move around at the beginning of the $19^{\text {th }}$ century, a shift of focus from wall to floor took place. Chairs and tables were arranged in the middle of the floor on a more regular basis, creating a "miniature internal landscape" that disengaged from the walls. ${ }^{7}$ The circular organization around a perimeter of a room changed to an intimate clustered occupation of the floor.

At this point one may begin to understand Evans' essay as an inquiry into the difficulties (and frustrations) of constructing a complete architectural representation of a room. For what happens to objects that are grouped on the floor when shown in a developed surface drawing? Should these objects be included in plan but omitted in the elevations of the walls? Or should they be included in all of the elevations? Or just one? And where does one position the sectional cuts through the interior needed for the elevations?

The eventual decline of the use of room portraits and their accompanying design principles can be understood as a loosening of the connection between the furnishings of an interior and its walls, floors and ceilings. Such a loosening can also become evident if an interior is examined after it has been inhabited. In the 1990s, Stewart Brand used "re-photography" - sequences of photographs of interiors captured from the same viewpoint over time - to make a case for how buildings, including their interiors, "learn" as time passes. ${ }^{8}$ Rather than depicting an ideal or intended state when furnished as anticipated by the designer, these photographs document a part of a room post occupancy, complete with everyday objects. A striking feature of most of the photographs is that the visual and spatial impact of objects and furniture, or "mobilia", often exceeds that of its architectural framework (Figure 3). The "learning" can be understood as a constant negotiation between the interior and its inhabitants, where both need to "shape and reshape themselves to each other until there's some kind of tolerable fit." The deployment of mobilia seems to be instrumental in finding the fit, and the photographs often document how objects are used beyond their intended function to open up new possibilities of inhabitation.

7. Ibid, 219.

8. S. Brand, How Buildings Learn: What Happens After They're Built (New York: Penguin Books, 1994), 212-213.

9. Ibid, 164. 

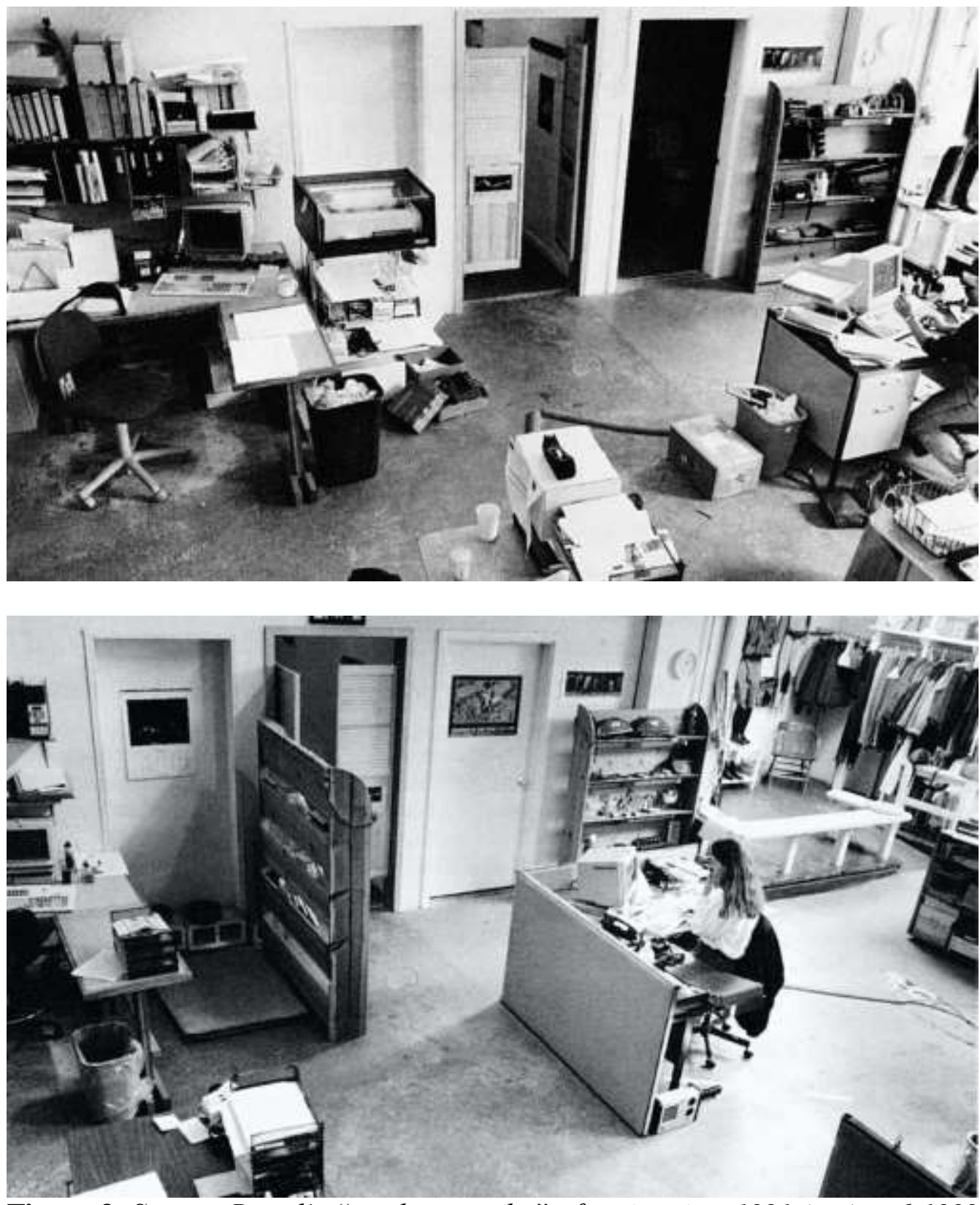

Figure 3. Stewart Brand's "re-photography" of an interior, 1991 (top) and 1992 (bottom). The study indicates the extent to which furniture and objects are rearranged over time.

Source: ( ) Stewart Brand.

\section{From Collection to Stuff}

There is a history of understanding the domestic interior through the inhabitants' belongings and how they are organized. In The Arcades Project, Walter Benjamin famously considered the emergence of the bourgeoise interior in 19th century Paris. During this time, industrial production replaced handicraft and 
objects became commodities that lacked the meaning that the bond between maker and user previously had given them. The act of bringing objects into one's home became a "task of divesting things of their commodity character by taking possession of them." $" 10$ The interior became the place in which a new kind of relation formed between a dweller and a collection of domesticated objects. The collection could testify to the fact that the dweller has had a particular experience and transport the mind back to this event. The objects in the collection thus became traces that could be read as a "detective story", allowing an observer an insight into the psyche of the dweller. ${ }^{11}$

In this way, Benjamin saw the interior as being produced in the process of inhabitation, where the interior begins as a void, an immaterial concept, that is subsequently defined materially by objects in it. This way of understanding the interior resonates with an account of the contemporary relation between the interior and its objects given by Sylvia Lavin in "Architecture in Extremis":

Stuff, things that have material lives, is not a secondary addition to the architectural situation, or merely an instrument of personalisation or functional fulfilment; rather it's what gives rise to architecture in the first place. The interior is produced not by walls and other boundaries but by the order, array, and number of objects within, which coalesce into a perceptible environment, not despite their generally varied and unpredictable presence, but by virtue of their objecthood independent of the envelope that contains them. ${ }^{12}$

Just as the word "stuff" is uncountable (a so-called mass noun), the stuff that fills our homes - furniture, books, clothes, houseware, textiles, waste, souvenirs, plants etc. - can be seen as an architectural entity, rather than as separate pieces. The "stuff" can sometimes be of modest amount and have little or subtle impact on the overall space of the interior. In other cases, belongings have been added, stored, and squeezed in, to the extent that they start to fill up the whole interior, claiming increasingly more of its space. The stuff might constrict the inhabitants to the point where it starts to impinge on their freedom or even become suffocating.

There is a threshold at which stuff becomes both a practical and architectural problem. Objects and furniture tend to be derogatively labelled "stuff" when they lack a designated place in the interior. Such a designated place has historically been provided by integrating an object into an overarching composition. Towards the end of the $19^{\text {th }}$ century, with the emergence of Jugendstil, for example, the architect became a total designer that worked across scales from the room to the small object. "The individuality expressed within the interior shifted from being that of the inhabitant, mediated through the collection, and became that of the architect-turned-artist, whose artistic "vision' constricted the inhabitant.",13

The stuff invading the modern domestic interior has partly been dealt with through the deployment of planned storage space. Alex O'Briant describes the

10. W. Benjamin, The Arcades Project (ed.) R. Tiedemann (trans.) H. Eiland and K. McLaughlin (Cambridge: Belknap Press of Harvard University Press, 2002), 9.

11. Ibid, 9, 20.

12. S. Lavin, “Architecture in Extremis," Log 22 (2011): 58.

13. Rice, The Emergence of the Interior: Architecture, Modernity, Domesticity (2007), 17. 
historical development of the closet, which can be said to originate from the residue spaces of $18^{\text {th }}$ century architecture, where the arrangement of geometrically designed rooms of rectangular, circular, hexagonal or elliptic shapes produced small spaces within the thickened walls, or poché, that turned out to be of practical use for storage. ${ }^{14}$ Closets, cupboards and other types of built-in domestic storage has since become a given in any home, holding and hiding the stuff dismissed from being on display in our living rooms or open plan kitchens. The required amount of storage space has increased successively during the last decades. Our stuff is no longer just filling up our closets, but also our basements, attics, garages, garden sheds and self-storage units.

The idea that stuff must either be kept in check by an overarching design sensibility or be kept from sight reveals an issue of authorship, as both approaches attempt to put the category of objects under the architect's control. Whether other approaches with a more relaxed grip on the whereabouts of objects can be considered as less "totalizing" and more flexible has been debated during the last decades. In the arguments of Mark Wigley, any attempt to conceive the interior as an "open" framework for the accommodation of objects that is more indeterminate or incomplete in nature can in itself be understood as a carefully authored aesthetic agenda that does not allow for interference. ${ }^{15}$ Andrew Holder has found this argument to simply be counterintuitive, while Michael Meredith has suggested that it may be possible to escape the totalizing ambition by arranging objects as if they were "campsites" - sites where objects are grouped and positioned without regard for an architectural framework. ${ }^{16}$ Whatever the case, embracing rather than shunning away from the fuzzy category of stuff might according to Sylvia Lavin open up new possibilities:

Imagining new definitions for architecture that include a bigger category of objects, a broader understanding of work, and that engage systems of instability and participation in the process of design no longer need to weaken architecture's cultural project but rather could make it more extreme. ${ }^{17}$

Together, these accounts of the objects in our homes suggest a shift from the idea of a collection, carefully organized by the dweller, to ever increasing amounts of stuff with agencies of its own. Today, space seems to be under constant siege by objects, and the ways in which these objects are used and placed often do not align with their intended purpose. In processes of being tucked away, displaced, or displayed, objects acquire their own lives that in their material and spatial organization redefine the architecture and produces a "live" interior. But despite

14. A. O’Briant, "Gray Space," Harvard Design Magazine, no.43 (2016): 90-97.

15. M. Wigley, "Whatever Happened to Total Design?" Harvard Design Magazine, no. 5 (1998): 1-8.

16. See A. Holder, "Whatever Happened to 'Whatever Happened to 'Whatever Happened to Total Design?'?"' Harvard Design Magazine, no. 47 (2019): 25-30; M. Meredith, "Whatever Happened to 'Whatever Happened to Total Design?'?: The Momentary Utopian Jouissance of the Bouroullec Brothers." Harvard Design Magazine, no. 29 (2008): 1-3.

17. Lavin, "Architecture in Extremis," (2011): 61. 
recent attempts, architecture may yet have to find a model for the interior that restores stuff as subject worthy of study and design consideration.

\section{Capturing the Live Interior}

The unattainable fantasy of an all-encompassing representation of an interior space that was established with the developed surface drawing has today been reincarnated in point clouds obtained with laser scanning. A survey of a space is no longer necessarily done through manual measuring and drawing notations complemented with photographs that capture qualities such as colour and texture. Laser scanning has folded these processes into one as each coloured point acquired with the scanner captures both the dimensions of the space as well as its phenomenal qualities. The scanner, unlike the eye of a human conducting a survey, does not discriminate between categories of objects or between what has been intentionally designed and what is circumstantial. It dutifully captures a space and all the objects contained in it in millions of points arrayed in a cartesian coordinate system.

The scanner's capacity to capture a space and everything in it has in an uncanny way actualized Benjamin's conceptualisation of the interior as a detective story. Eyal and Ines Weizman, in collaboration with ScanLAB, have used 3Dscanning towards such forensic ends. The work of these practices implies that a scene captured with scanning can be understood as a scenario, a frozen moment in time in which the relation between objects captured begins to unfold a narrative. The diffusion of scanning for the purposes surveying everything from crime scenes to landscapes means that the world is increasingly reconstructed at levels of detail that may constitute an "invasion of privacy." 18

Interiors Matter: A Live Interior explores how interiors are produced by ongoing arrangement and accumulation of belongings, using point clouds obtained with laser scanning as a means of architectural representation. In the first phase of the project, documented in this paper, inhabited interiors in two early to midcentury apartment buildings in Stockholm were documented during 2019. Both buildings contain rentals, implying that rooms and apartments to a large extent are comparable with regards to space plan and finishes. This means that the imprint made by each tenant consists mostly of their furniture and belongings. Although separated by some 20 years (1929 and 1946), the studied apartments in both buildings shared a few characteristics. They were all relatively small - studios or one-bedroom apartments - and they all featured rooms that in their size and space plan did not prescribe a particular layout of furniture.

Rooms in the apartments were documented using laser scanning, also known as LiDAR (Light Detection and Ranging), a method that uses light to measure distances to surfaces that a scanner can "see" from a fixed point in space. The scanner emits a short pulse of light and times how long it takes for the pulse to be

18. M. Shaw and W. Trossell, "Digital Doppelgängers: Future Scanscapes," Architectural Design 84, no. 1, (2014): 22. 
reflected back in order to estimate depth to a point on an object in space. ${ }^{19}$ Each pulse results in a point in a coordinate system and many points together form a point cloud. A scanned interior is constructed as a thin crust of points that envelopes the room by clinging to floor, walls, and ceiling, as well as furniture and objects.

The process of acquisition is based on a logic of assembly of data from a series of rotational scans from different positions, referred to as stations. For each station, a camera with a fisheye lens was used to capture the room in six photographs that were subsequently "stitched" together to a 360-degree panorama. The panorama was projected spherically onto the point cloud in order to give each colourless point in the cloud an RGB colour value. The scanning technique is both spatial and temporal, as each station produces a point cloud specific to its position at a given moment in time. A certain station will provide a clear reading of some surfaces belonging to an interior, while other surfaces will be obstructed from view. Point clouds from several stations are registered and assembled into one in order to get a fairly complete reading of the space and the objects contained in it. The combined cloud is therefore the result of several spatial and temporal recordings. The point clouds were used to produce a series of representations, referred to as cloud drawings.

\section{Cloud Drawings}

Light is pouring through a window, dissolving the white curtain against the wall. A carpet with a small table atop sits in front of the window in the centre of the room. To the left, a grey, plush sofa and a floor lamp with a frilly beige shade. A wooden cabinet supporting a small green vase with red flowers is visible behind the sofa. At the right side of the table stands a rattan armchair with white upholstery and further to the right, against the wall, a wooden chiffonier. Below the chiffonier and armchair piles of books, boxes and unidentifiable objects are dimly seen. Two lampshades seem to levitate above the floor-bound furniture.

This mundane domestic scenery is captured in one of the cloud drawings (Figure 1). As a representation, it bears resemblance to a pointillist painting, or a grainy photograph, while at the same time complying to the conventions of cut and elevation native to an architectural section drawing. It is a recording of a domestic interior from within, captured in 800 million coloured points - a documentation of the framework of the space as well as all objects contained in it. In this matrix of points there are gaps where surfaces are missing behind and below objects. The curtain and its rod cast a white shadow on the wall, objects under the armchair seem to dissolve into a thin mist, and the interior of the chiffonier is missing entirely. These are spots that the beam of the laser scanner has not been able to reach.

When explored through a modelling interface, the point cloud in its unedited state may at first seem incomprehensible and partly impenetrable. Each room, or

19. For a brief summary of the principles of LiDAR, see for example M. Gross and H. Pfister, Point-Based Graphics (ed.) H. Pfister (Elsevier Science \& Technology, 2007), 38. 
sequence of rooms, appears as a positive object formed by negative space. It resembles a cast of the void space of the room, a comprehension of space that has been popularized by artist Rachel Whiteread's casts such as Ghost (1990). This often nearly closed volume (Figure 4) needs to be clipped with a plane in order to get views of the room from the inside. Most of the cloud drawings presented here explore clipping as a way to reveal different aspects of the interior and the relation between the architectural framework and the objects of the interior.

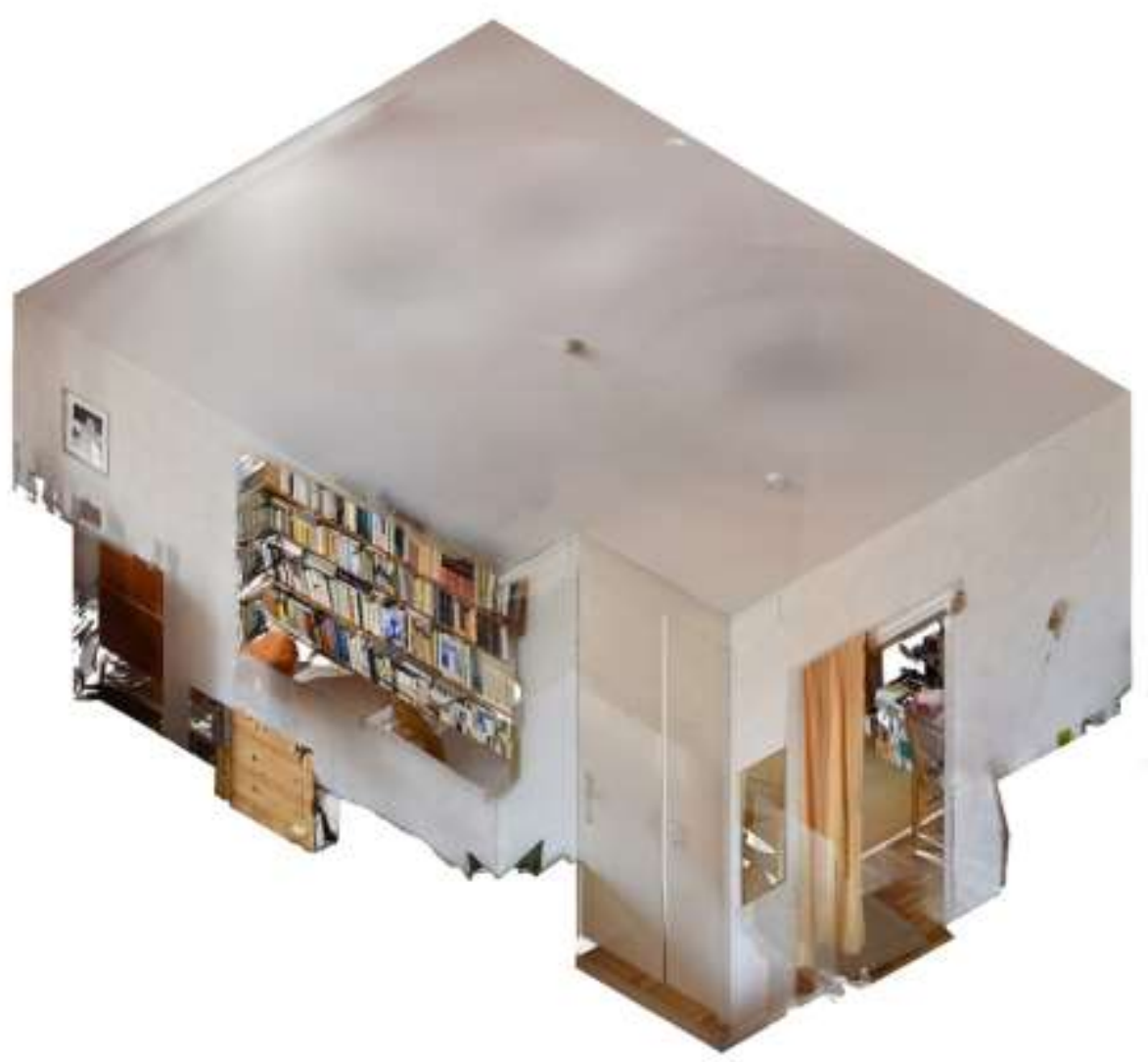

Figure 4. Interiors Matter: A Live Interior. Isometric view of unedited point cloud acquired through laser scanning a room in an apartment, Stockholm, 2019. The room appears as a positive cast of negative space.

Source: Authors.

Some of the scanned rooms were crowded with stuff, almost as if walls and floors had become encrusted with furniture and objects. In one set of drawings the walls, floor, and ceiling of such a room were clipped away (Figure 5). This meant that the entire room could be captured in an exterior elevation - as if it was an aquarium - that compresses the depth of the interior into layers of objects, revealing the extent to which the shape of the room can be defined by the stuff that it contains. Surfaces in the foreground, such as those belonging to a sofa, framed pictures, a corner cupboard, and stack of books, are viewed from behind (hence the mirrored letters), while objects further away from the viewing plane appear as one would expect them to in a typical elevation. The level of detail sustains at least 
two modes of attention: a more distanced viewing where the room is defined by a jumble of distinct objects, and a more myopic view that reveals minute details in each object, as well as messy parts of the point cloud where objects become indistinct due to a lack of resolution.

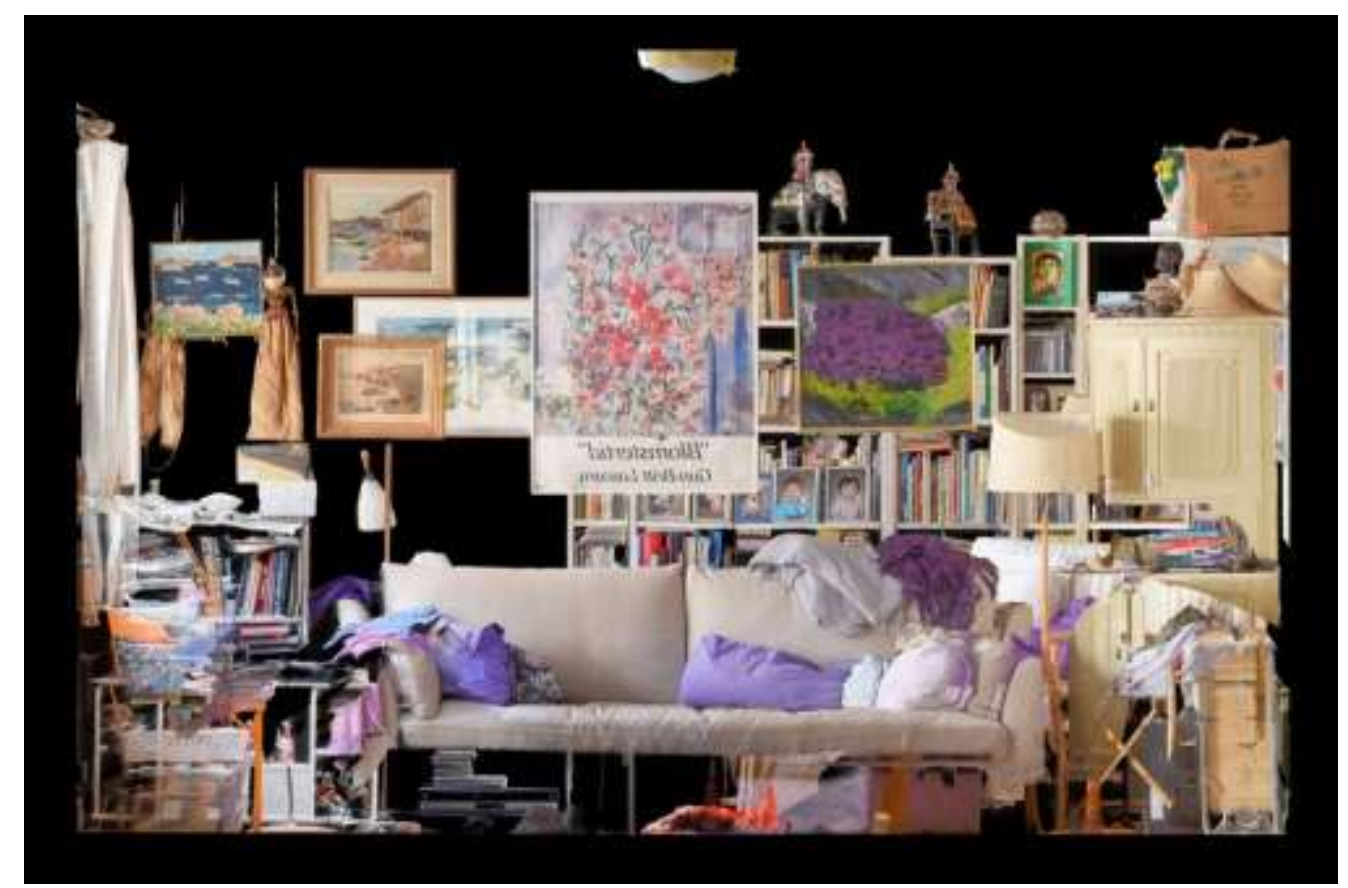

Figure 5. Interiors Matter: A Live Interior. Exterior view of point cloud acquired through laser scanning of a room in an apartment, Stockholm, 2019. Floor, walls, and ceiling have been clipped away, revealing the extent to which the interior is shaped by furniture and objects.

Source: Authors.

The temporal aspect of cloud drawing was explored in an animated drawing that focused on the changing depiction of a flower vase when captured from different stations (Figure 6). A series of still images from the animation show how the level of detail and densities of points change with the position of the scanner. As the viewing angle to the chiffonier and the flower varies, certain parts of objects were captured and others blocked out, producing holes in the cloud shown as white areas in the render. Due to the varying scanner positions, shifting light conditions as well as slight movement, the vase and the flowers appear as ghostly, flickering objects. 


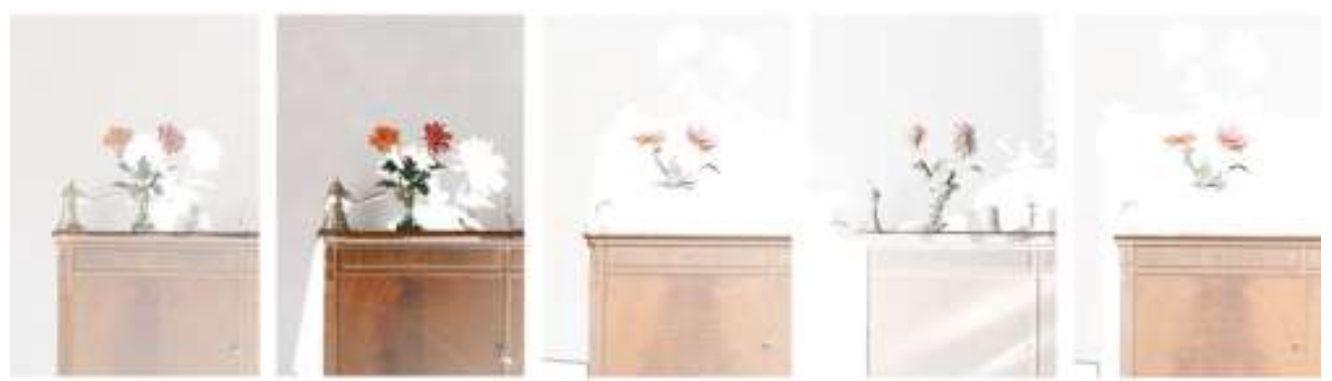

Figure 6. Interiors Matter: A Live Interior. Detail view of point cloud acquired through laser scanning of a room in an apartment, Stockholm, 2019. A series of still images from an animated cloud drawing show how the level of detail and densities of points change with the position of the scanner. Source: Authors.

There are a number of representational discrepancies between the point cloud and other more common ways of representing architecture. Traditional architectural drawings are constructed of lines, curves and edges that unambiguously define the limits of objects. Cloud drawings, in contrast, consist solely of points and can only illusively produce the reading of a line when multiple points share one coordinate value in a plane. Even a relatively sharp edge in a cloud drawing carries a depth due to the cloud's multilayering of data from different stations. The cloud drawing, especially when clipped to a short depth, suggests a continuity across objects (Figure 7).

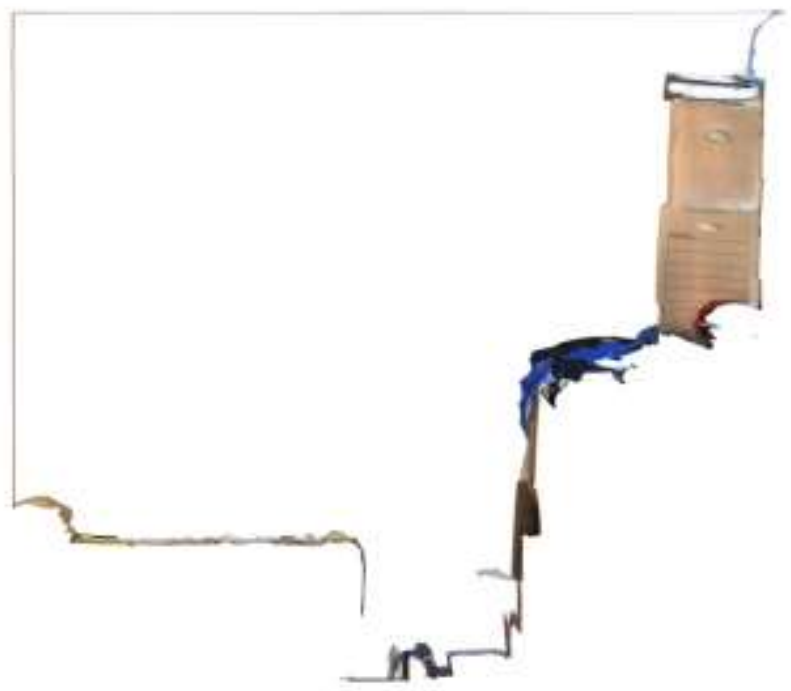

Figure 7. Interiors Matter: A Live Interior. Section of point cloud acquired through laser scanning of a room in an apartment, Stockholm, 2019. The section has been clipped to a short depth, suggesting a continuity between wall, ceiling, and a temporary arrangement of objects.

Source: Authors.

The acquired point clouds appear as complete, high fidelity representations of a room, while at the same time being limited and fragmented. The scanner can 
only capture a surface or an object that it gets an unobstructed view of. Deep recesses or crevices between tightly grouped objects become shadowy areas of missing information. A surface of an object that faces a wall or another object is typically not possible to capture. The point cloud measures visible objects as well as the spots that the scanner cannot see, and these defects are prominently featured even when one does clip the cloud to a view or projection. One set of drawings directs attention to the parts of the room that are invisible from a single station (Figures 1, 6). These drawings feature whiteout areas, such as a "drop shadow" of a vase and a curtain rod on the elevation of the back wall.

It is revealing to compare the effects of the cloud drawings with those of the developed surface drawing technique described by Evans, which provided a certain unification of the interior including drapes, furnishings, wall coverings, objects, etc. The developed surface drawing had a flattening effect on the architecture that it represented that is distinct from the sense of depth and threedimensionality of a cloud drawing. But both modes of representation have a unifying effect as they encapsulate the void of a room, including the perimeter with its furnishings as well as furniture and objects. The developed surface technique affected the design of furniture, as the backsides of chairs and sofas facing a wall often were left undecorated or painted white. Just like the blind spots that appear in a point cloud acquired with laser scanning, these backsides are surfaces that elude both documentation and display (Figures 1, 6, 7).

\section{Environments, Assemblies, Materialities}

As much as the cloud drawings explore the representational particularities of point clouds, they are ultimately a documentation of domestic scenes. The process of editing and formatting the point clouds to arrive at a set of drawings focussed on three ways of reading a scene, through environments, assemblies, and materialities. Together, these notions became a way to discern and conceptualize qualities in drawings as worked progressed. They loosely suggest shifts in scale, from the room or environment, via assemblies of objects, to the materiality of single objects. At the same time, they constitute a way of reading the interior that goes beyond a familiar parsing according to scale. Zooming and clipping might for example focus a drawing on an environment surrounding a single object in a corner of a room.

An environment amounts to the circumstances, objects, or conditions by which something is surrounded. Some of the cloud drawings downplay the importance of individual objects in favour of focusing on an environment as a whole. They direct attention to the ways in which the larger figure of the room is defined by ceiling, walls and floor, as well as by furniture and objects (Figure 1). Stuff may be omnipresent throughout the room in these drawings, but the view and the clipping plane of the section has been selected to suggest a larger environment. The distinction between architecture, furniture, and smaller objects found in a conventional architectural drawing start to break down. The void of the room is as effectively shaped by the distinct corner of ceiling and wall, as it is by a fuzzier 
mass consisting of a bookshelf, a chaise longue, pillows and blankets, a porcelain urn, that seem to have been smeared across the intersection between the wall and the floor (Figure 8). As objects lining the periphery of the room obscure the view of the scanner, they begin to replace floor, walls and ceiling as the delimitation of the room. In some instances, a room is largely defined by an inner layer of surfaces that leave a void in-between object and architecture. These leftover spaces can be characterized as smaller and sometimes blurrier versions of Robert Venturi's notion of "residual spaces", a more or less open layer of surfaces that together shape a space in an interior. ${ }^{20}$

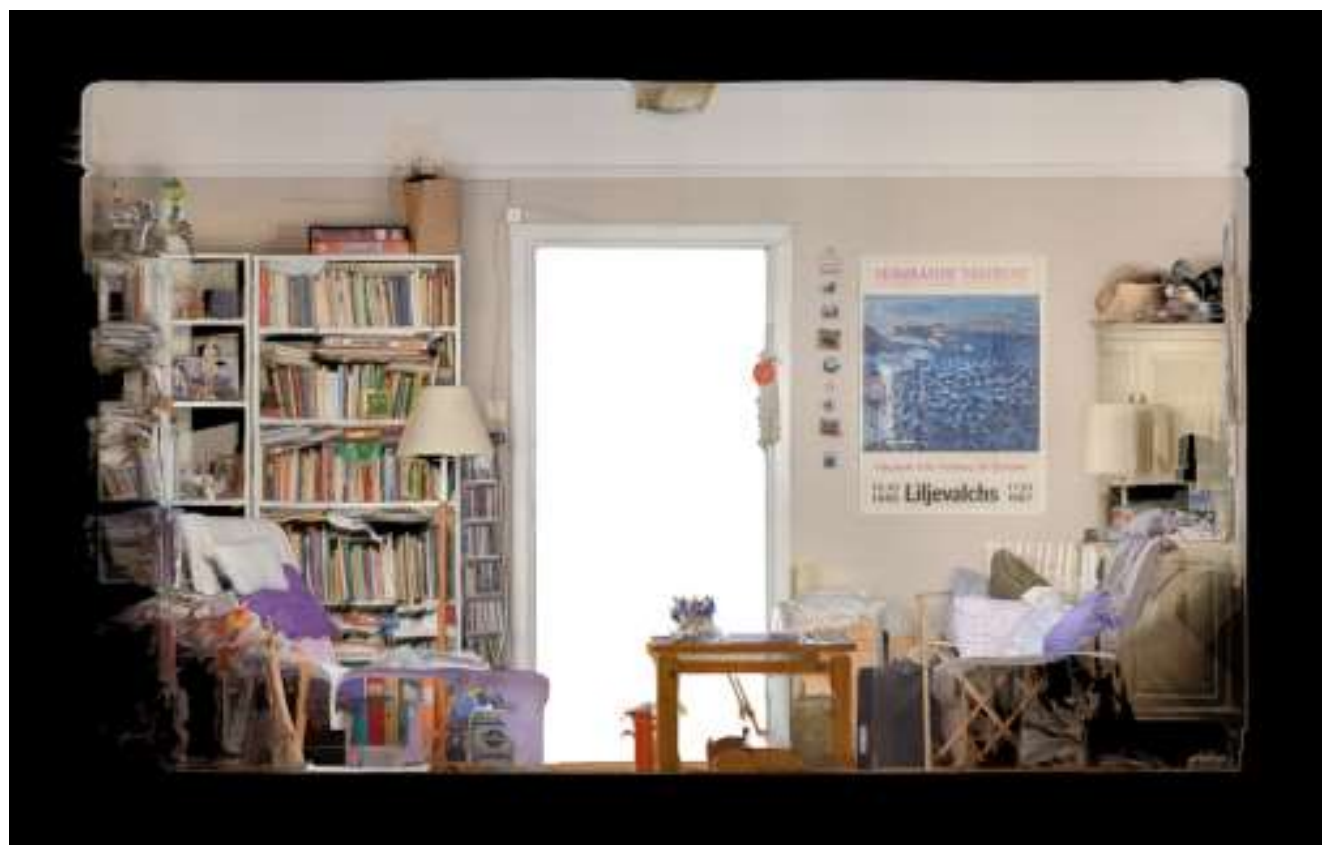

Figure 8. Interiors Matter: A Live Interior. Section of point cloud acquired through laser scanning of a room in an apartment, Stockholm, 2019. As objects lining the periphery of the room obscure the view of the scanner, they begin to replace floor, walls and ceiling as the delimitation of the room. Source: Authors.

Particular arrangements of furniture and objects can be identified as assemblies. This reading reveals hierarchies, adjacencies and interdependencies among domestic items and habits. Assemblies can be highlighted by clipping the point cloud so that the section cut reveals local continuity between two or several objects. This might, for example, allow us to understand "the book and the shelf that it sits on", or similarly "the pillow and the sofa that it sits on", as an assembly and as a new whole, and ultimately as an architectural entity. In some cases, an assembly might be a particular situation of interest. When placed next to lounge chair, a pile of books with a glass on top might together be understood as something else, more similar to a small table. In this way, assemblies may shift

20. R. Venturi, "The Inside and the Outside," in Complexity and Contradiction in Architecture (New York: The Museum of Modern Art, 1966). 
qualities and habits of use associated with one object towards a new, larger entity composed of several objects. Several assemblies were documented in isometric view and compiled in a matrix (Figure 9). Through clipping planes in the $\mathrm{x}, \mathrm{y}$, and $\mathrm{z}$-axis, each assembly emerges as a local mies en scène, formed by walls, floor, or ceiling, as well as by furniture and objects. Some assemblies centre on an inbetween space defined by distinct objects, while others emphasise agglomerations of stuff, such as how a myriad of objects on a table define miniature topography.
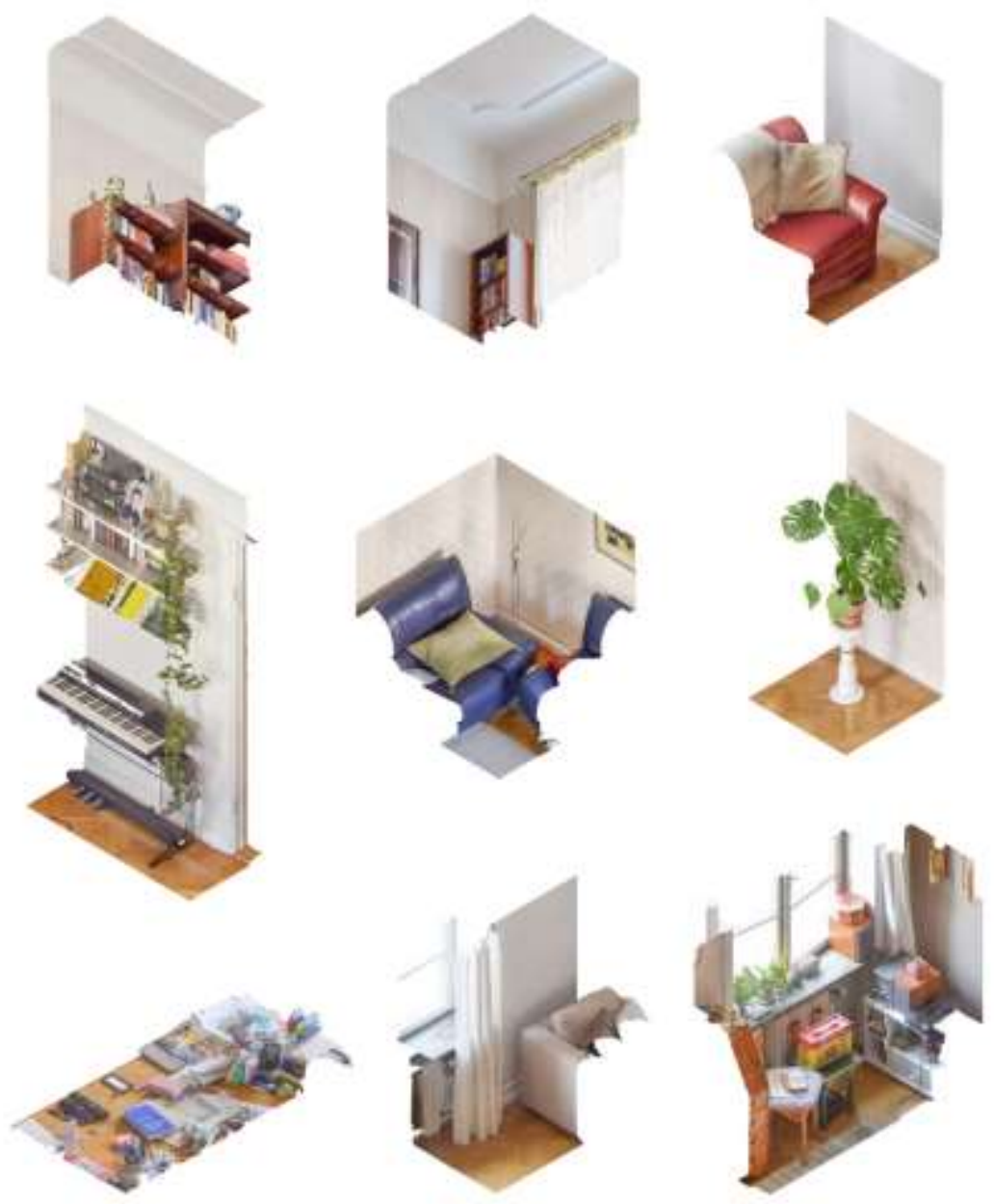

Figure 9. Interiors Matter: A Live Interior. Detailed isometric views of point cloud acquired through laser scanning of a room in an apartment, Stockholm, 2019. Each view has been clipped in order to reveal local assemblies of furniture and objects.

Source: Authors.

Materialities, finally, are considered as the material qualities belonging to an object, such as texture and colour, or highlight and shadow. In the cloud drawings, materialities range from realistic depictions of material effects, to glitches and spots of missing information in the point cloud. A clipped and zoomed-in view of the corner of a sofa, for example, features the subtle shifts in tone belonging to 
wrinkles and stitches on the upholstery (Figure 10). In addition, it reveals the stippled effect of missing points and adjacent points of contrasting colour. The materiality shifts between moments where the medium of the point cloud becomes invisible and other moments where the materiality is highly conditioned by the medium. This is especially true for materials that are reflective or diaphanous in nature. Reflective or transparent materials, such as shiny metals, glass or water will many times elude acquisition because of laser beams being reflected or refracted. The presence of such materials might instead produce disturbances or glitches in nearby areas. The surface of a mirror will reflect the light pulse, so that it appears as if it was an opening to another space, while a thin curtain might disappear momentarily as the laser passes through it. Further, a draught through a room may gently shift the position of lightweight objects that are suspended in the space.

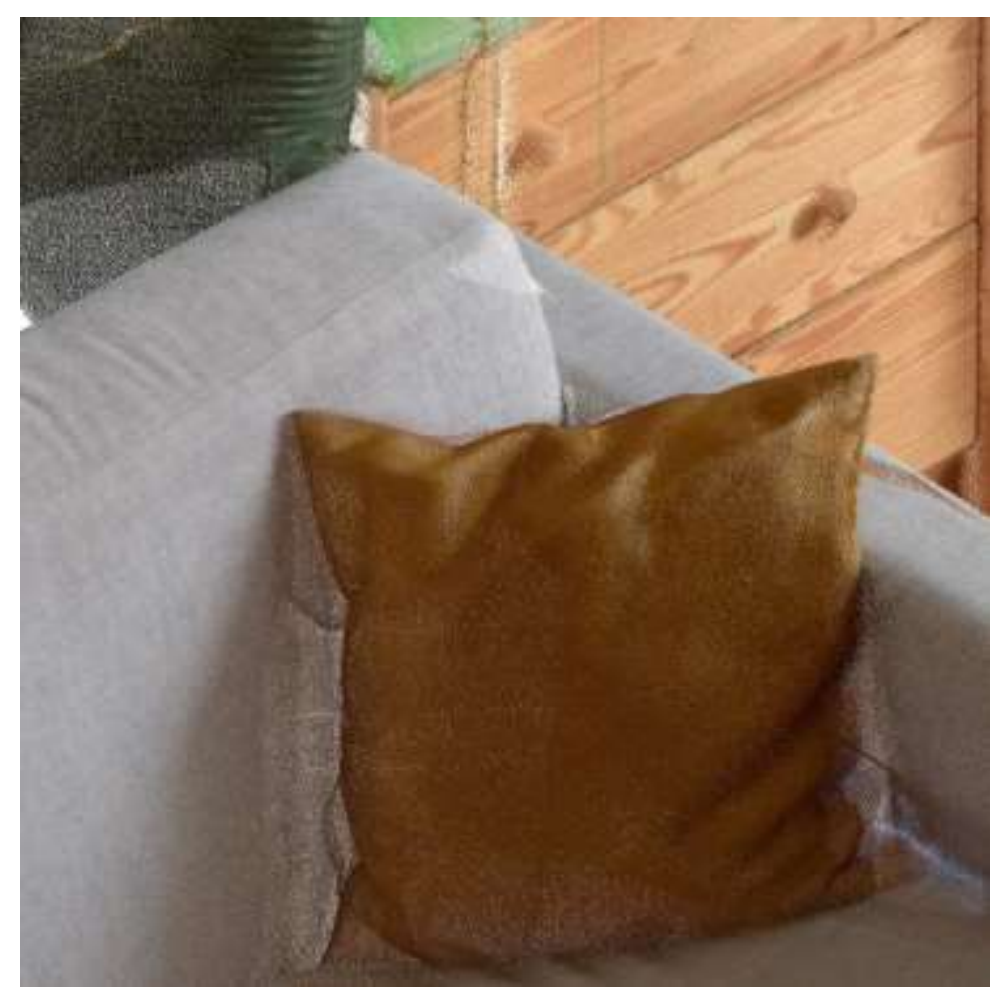

Figure 10. Interiors Matter: A Live Interior. Detailed isometric views of point cloud acquired through laser scanning of a room in an apartment, Stockholm, 2019. Different materialities are mediated through the point cloud, such as the softness of the pillowcase and the wooden grain of the chest of drawers. Source: Authors.

The three categories can be used to study effects at different scales but can also be considered simultaneously to describe parallel or layered qualities. 


\section{Conclusions}

If there is a reciprocal influence between techniques for representing interiors and the development of spatial practice, how may cloud drawings possibly contribute to new notions of what constitutes an interior? And if the relation between architectural delineation and the transitory, provisional placement of stuff historically has shifted between loose and fixed - how can we understand this reciprocity today?

Envisioning new categories for the interior such as environments, assemblies and materialities is an alternative to a more conventional parsing based on different scales or design disciplines. The separation of an interior into architecture, furniture, and objects and further into different professional responsibilities can be a missed opportunity to rethink the interior. This separation, along with the difficulties of representing a space and the artefacts contained in it, may be two reasons why the interior remains an unstable and shifting concept.

In order to be able to address systems of instability of domestic interiors, it is first necessary to be able to document them. Coupling techniques of laser scanning and the processing of point clouds with architectural techniques of representation through elevations, sections and isometric views, the Interiors Matter project attempts to uncover new ways of understanding and conceptualizing the interior. Adopting the scanner's way of seeing, where no distinction is made between scales or types, between the room and the candlestick, makes it possible to assign equal weight to architectural framework, furniture and objects. Everything that stands in the way of the scanner gets surveyed, ignoring disciplinary hierarchies. This mode of documentation puts focus on the informal qualities of a domestic interior, qualities that most likely would go unnoticed if not recorded by the $3 \mathrm{~d}$ scanning.

The cloud drawings presented here reveal new dependencies as well as informal and temporal relationships by cutting through loosely arranged interior belongings. These drawings amount to a proposition for the interior that recasts the messy category and the influential agencies of stuff as an architectural entity.

\section{Acknowledgments}

Interiors Matter: A Live Interior is a three-year artistic research project funded by The Swedish Research Council and hosted by KTH Royal Institute of Technology and Konstfack, College of Arts, Crafts and Design in Stockholm.

\section{Bibliography}

Aureli, P. V. Life, Abstracted: Notes on the Floor Plan. E-flux Architecture, 2017. Retrieved from: https://www.e-flux.com/architecture/representation/159199/life-abs tracted-notes-on-the-floor-plan/. [Accessed 26 October 2020]

Benjamin, W. The Arcades Project. Edited by R. Tiedemann. Translated by H. Eiland and K. McLaughlin. Cambridge: Belknap Press of Harvard University Press, 2002. 
Brand, S. How Buildings Learn: What Happens After They're Built. New York: Penguin Books, 1994.

Evans, R. "The Developed Surface: An Enquiry into the Brief Life of an EighteenthCentury Drawing Technique." In Translations from Drawing to Building, 194-231. Cambridge: The MIT Press, [1989] 1997.

Gross, M. and H. Pfister. Point-Based Graphics. Edited by H. Pfister. Elsevier Science \& Technology, 2007.

Holder, A. "Whatever Happened to 'Whatever Happened to 'Whatever Happened to Total Design?'?" Harvard Design Magazine, no. 47 (2019): 25-30.

Lavin, S. “Architecture in Extremis.” Log 22 (2011): 51-61.

Meredith, M. "Whatever Happened to 'Whatever Happened to Total Design?'?: The Momentary Utopian Jouissance of the Bouroullec Brothers." Harvard Design Magazine, no. 29 (2008): 1-3.

O’Briant, A. “Gray Space.” Harvard Design Magazine, no.43 (2016): 90-97.

Rice, C. The Emergence of the Interior: Architecture, Modernity, Domesticity. London and New York: Routledge, 2007.

Shaw, M. and W. Trossell. "Digital Doppelgängers: Future Scanscapes." Architectural Design 84, no. 1, (2014): 20-29.

Venturi, Robert. "The Inside and the Outside." In Complexity and Contradiction in Architecture, 70-87. New York: The Museum of Modern Art, 1966.

Wigley, M. "Whatever Happened to Total Design?" Harvard Design Magazine, no. 5 (1998): 1-8. 
